\title{
Algebraic Shifting and Basic Constructions on Simplicial Complexes
}

ERAN NEVO

eranevo@math.huji.ac.il

Institute of Mathematics, The Hebrew University, Givat Ram, Jerusalem 91904, Israel

Received April 1, 2003; Revised January 10, 2005; Accepted May 6, 2005

\begin{abstract}
We try to understand the behavior of algebraic shifting with respect to some basic constructions on simplicial complexes, such as union, coning, and (more generally) join. In particular, for the disjoint union of simplicial complexes we prove $\Delta(K \cup \dot{\cup})=\Delta(\Delta(K) \dot{\cup} \Delta(L))$ (conjectured by Kalai [6]), and for the join we give an example of simplicial complexes $K$ and $L$ for which $\Delta(K * L) \neq \Delta(\Delta(K) * \Delta(L))$ (disproving a conjecture by Kalai [6]), where $\Delta$ denotes the (exterior) algebraic shifting operator. We develop a 'homological' point of view on algebraic shifting which is used throughout this work.
\end{abstract}

Keywords: algebraic shifting, simplicial complexes

\section{Introduction}

Algebraic shifting is an operator which associates with each simplicial complex another simplicial complex which is combinatorially simpler (is shifted: to be defined shortly) and preserves some properties of the original complex. It was introduced by Kalai [2].

In this work we try to understand the behavior of algebraic shifting with respect to some basic constructions on simplicial complexes, such as union, cone, and (more generally) join. In some cases we get a 'nice' behavior: We prove that the disjoint union of simplicial complexes satisfies

$$
\Delta(K \dot{\cup} L)=\Delta(\Delta(K) \dot{\cup} \Delta(L))
$$

(conjectured by Kalai [6]). Moreover, we give an explicit combinatorial description of $\Delta(K \dot{\cup} L)$ in terms of $\Delta(K)$ and $\Delta(L)$. These results follow from the following theorem about shifting a (not necessarily disjoint) union: Define $\operatorname{init}_{j}(S)$ to be the set of lexicographically least $j$ elements in $S$, for $S \subseteq \mathbb{N}$, where $\mathbb{N}$ is the set of positive integers endowed with the usual order. For every $i>0$ define $I_{S}^{i}=I_{S}^{i}(n)=\{T: T \subseteq[n],|T|=$ $\left.|S|+i, \operatorname{init}_{|S|}(T)=S\right\}$. Denote by $K_{i}$ the $i$-th skeleton of a simplicial complex $K$.

Theorem 1.1 Let $K$ and $L$ be two simplicial complexes, and let $d$ be the dimension of $K \cap L$. For every subset $A$ of the set of vertices $[n]=(K \cup L)_{0}$, the following additive formula holds:

$$
\left|I_{A}^{d+2} \cap \Delta(K \cup L)\right|=\left|I_{A}^{d+2} \cap \Delta(K)\right|+\left|I_{A}^{d+2} \cap \Delta(L)\right|
$$


In the case where $K \cap L$ is a simplex (with all of its subsets), the following stronger assertion holds:

Theorem 1.2 Let $K$ and $L$ be two simplicial complexes, where $K \cap L=\langle\sigma\rangle$ is the simplicial complex consisting of the set $\sigma$ and all of its subsets. For every $i>0$ and every subset $S$ of the set of vertices $[n]=(K \cup L)_{0}$, the following additive formula holds:

$$
\left|I_{S}^{i} \cap \Delta\left(K \cup_{\sigma} L\right)\right|=\left|I_{S}^{i} \cap \Delta(K)\right|+\left|I_{S}^{i} \cap \Delta(L)\right|-\left|I_{S}^{i} \cap 2^{[|\sigma|]}\right| .
$$

This theorem gives an explicit combinatorial description of $\Delta(K \cup L)$ in terms of $\Delta(K)$, $\Delta(L)$ and $\operatorname{dim}(\sigma)$. In particular, any gluing of $K$ and $L$ along a $d$-simplex results in the same shifted complex $\Delta(K \cup L)$, depending only on $\Delta(K), \Delta(L)$ and $d$.

Another instance of a 'nice' behavior is

$$
\Delta \circ \text { Cone }=\text { Cone } \circ \Delta
$$

(Kalai [6]). We prove a generalized version of this property for near-cones (defined in [1]). In the case of join, we do not get such a good behavior: We give an example of simplicial complexes $K$ and $L$ for which

$$
\Delta(K * L) \neq \Delta(\Delta(K) * \Delta(L))
$$

where $*$ denotes the join operator, disproving a conjecture by Kalai [6]. However, a weaker assertion holds:

Theorem 1.3 Let $K$ and $L$ be two simplicial complexes with $\left|(K * L)_{0}\right|=n$. Then for every $i \in[n]$ :

$$
\begin{aligned}
|\{S \in \Delta(K * L):[i] \cap S=\emptyset,|S|=\operatorname{dim}(K * L)+1\}| \\
=|\{S \in \Delta(K):[i] \cap S=\emptyset,|S|=\operatorname{dim}(K)+1\}| \\
\quad \times|\{S \in \Delta(L):[i] \cap S=\emptyset,|S|=\operatorname{dim}(L)+1\}| .
\end{aligned}
$$

The case $i=1$ was known — it follows from the Künneth theorem with field coefficients [7] and from the combinatorial interpretation of homology using shifting (Björner and Kalai [1]). We give an example that Theorem 1.3 does not hold for symmetric shifting.

For a survey on algebraic shifting, the reader can consult [6].

Outline: Section 2 introduces the needed algebraic background. Section 3 develops a 'homological' point of view on the algebraic shifting operator which will be used in the successive sections. Section 4 shifts unions of simplicial complexes (proving Theorems 1.1 and 1.2), Section 5 shifts near-cones, Section 6 shifts joins of simplicial complexes (proving Theorem 1.3). 


\section{Algebraic background}

In this section we set some needed preliminaries. We follow the definitions and notation of [6]. Let $K$ be a simplicial complex on a vertex set [n]. The $i$-th skeleton of $K$ is $K_{i}=$ $\{S \in K:|S|=i+1\}$. For each $1 \leq k \leq n$ let $<_{L}$ be the lexicographic order on $\left(\begin{array}{c}{[n]} \\ k\end{array}\right)$, i.e. $S<_{L} T \Leftrightarrow \min \{a: a \in S \triangle T\} \in S$, and let $<_{P}$ be the partial order defined as follows: Let $S=\left\{s_{1}<\cdots<s_{k}\right\}, T=\left\{t_{1}<\cdots<t_{k}\right\}, S<_{P} T$ iff $s_{i} \leq t_{i}$ for every $1 \leq i \leq k$ (min and $\leq$ are taken with respect to the usual order on $\mathbb{N}$ ). $K$ is called shifted if $S<_{P} T$ and $T \in K$ implies $S \in K$.

Let $V$ be an $n$-dimensional vector space over $\mathbb{R}$ with basis $\left\{e_{1}, \ldots, e_{n}\right\}$. Let $\wedge V$ be the graded exterior algebra over $V$. Denote $e_{S}=e_{s_{1}} \wedge \ldots \wedge e_{s_{j}}$ where $S=\left\{s_{1}<\cdots<s_{j}\right\}$. Then $\left\{e_{S}: S \in\left(\begin{array}{c}{[n]} \\ j\end{array}\right)\right\}$ is a basis for $\bigwedge^{j} V$. Note that as $K$ is a simplicial complex, the ideal $\left(e_{S}: S \notin K\right)$ of $\bigwedge V$ and the vector subspace $\operatorname{span}\left\{e_{S}: S \notin K\right\}$ of $\bigwedge V$ consist of the same set of elements in $\bigwedge V$. Define the exterior algebra of $K$ by

$$
\bigwedge(K)=(\bigwedge V) /\left(e_{S}: S \notin K\right) \text {. }
$$

Let $\left\{f_{1}, \ldots, f_{n}\right\}$ be a basis of $V$, generic over $\mathbb{Q}$ with respect to $\left\{e_{1}, \ldots, e_{n}\right\}$, which means that the entries of the corresponding transition matrix $A\left(e_{i} A=f_{i}\right.$ for all $\left.i\right)$ are algebraically independent over $\mathbb{Q}$. Let $\tilde{f}_{S}$ be the image of $f_{S} \in \bigwedge V$ in $\bigwedge(K)$. Define

$$
\Delta(K)=\Delta_{A}(K)=\left\{S: \tilde{f}_{S} \notin \operatorname{span}\left\{\tilde{f}_{S^{\prime}}: S^{\prime}<_{L} S\right\}\right\}
$$

to be the shifted complex (introduced by Kalai [2]). The construction is canonical, i.e. it is independent of the choice of the generic matrix $A$, and for a permutation $\pi:[n] \rightarrow[n]$ the induced simplicial complex $\pi(K)$ satisfies $\Delta(\pi(K))=\Delta(K)$. It results in a shifted simplicial complex, having the same face vector and Betti vector as $K$ 's [1] (for a simplicial complex $L$, its face vector $f(L)=\left(f_{i}(L)\right)$ is defined by $f_{i}(L)=\left|L_{i}\right|$, and its Betti (homology) vector $\beta(L)=\left(\beta_{i}(L)\right)$ is defined by $\beta_{i}(L)=\operatorname{dim} \tilde{H}_{i}(L, \mathbb{R})$, where $\tilde{H}_{i}()$ stands for the reduced $i$-th homology). The key ingredient in Björner and Kalai's proof that algebraic shifting preserves Betti numbers, is the following combinatorial way of reading them:

$$
\beta_{i}(L)=\left|\left\{S \in \Delta(L)_{i}: S \cup 1 \notin \Delta(L)\right\}\right|
$$

Fixing the basis $\left\{e_{1}, \ldots, e_{n}\right\}$ of $V$ induces the basis $\left\{e_{S}: S \subseteq[n]\right\}$ of $\bigwedge V$, which in turn induces the dual basis $\left\{e_{T}^{*}: T \subseteq[n]\right\}$ of $(\bigwedge V)^{*}$ by defining $e_{T}^{*}\left(e_{S}\right)=\delta_{T, S} .\left((\bigwedge V)^{*}\right.$ stands for the space of $\mathbb{R}$-linear functionals on $\bigwedge V$.) For $f, g \in \bigwedge V<f, g>$ will denote $f^{*}(g)$. Define the so called left interior product of $g$ on $f$ [3], where $g, f \in \wedge V$, denoted $g\lfloor f$, by the requirement that for all $h \in \bigwedge V$

$$
<h, g\lfloor f>=<h \wedge g, f>\text {. }
$$

( $g\lfloor\cdot$ is the adjoint operator of $\cdot \wedge g$ w.r.t. the inner product $<\cdot, \cdot>$ on $\bigwedge V$.) Thus, $g\lfloor f$ is a 
bilinear function, satisfying

$$
e_{T}\left\lfloor e_{S}= \begin{cases}(-1)^{a(T, S)} e_{S \backslash T} & \text { if } T \subseteq S \\ 0 & \text { otherwise }\end{cases}\right.
$$

where $a(T, S)=|\{(s, t) \in S \times T: s \notin T, t<s\}|$. This implies in particular that for a monomial $g$ (i.e. $g$ is a wedge product of elements of degree 1) $g\lfloor$ is a boundary operation on $\bigwedge V$, and in particular on $\operatorname{span}\left\{e_{S}: S \in K\right\}[3]$.

Let us denote for short $\bigwedge^{j} K=\operatorname{span}\left\{e_{S}: S \in K_{j-1}\right\}$ and $\bigwedge K=\bigwedge(K)=\operatorname{span}\left\{e_{S}:\right.$ $S \in K$ \}. This should cause no confusion with the definition of the exterior algebra of $K$, as we shall never use this exterior (quotient) algebra structure in the sequel, but only the graded vector space structure $\bigwedge K$ just defined. We denote:

$$
\operatorname{Ker}_{j} f_{R}\left\llcorner(K)=\operatorname{Ker}_{j} f_{R}\left\llcorner=\operatorname{Ker}\left(f_{R}\left\llcorner: \bigwedge^{j+1} K \rightarrow \bigwedge^{j+1-|R|} K\right)\right.\right.\right.
$$

Note that the definition of $\bigwedge K$ makes sense more generally when $K_{0} \subseteq[n]$ (and not merely when $\left.K_{0}=[n]\right)$, and still $f_{R} L$ operates on the subspace $\bigwedge K$ of $\bigwedge V$ for every $R \subseteq[n]$. (Recall that $f_{i}=\alpha_{i 1} e_{1}+\cdots+\alpha_{i n} e_{n}$ where $A=\left(\alpha_{i j}\right)_{1<i, j \leq n}$ is a generic matrix.) Define $f_{i}^{0}=\sum_{j \in K_{0}} \alpha_{i j} e_{j}$, and $f_{S}^{0}=f_{s_{1}}^{0} \wedge \cdots \wedge f_{s_{j}}^{0}$ where $S=\left\{s_{1}<\cdots<s_{j}\right\}$. By Eq. (3), the following equality of operators on $\bigwedge K$ holds:

$$
\forall S \subseteq[n] f_{S}\left\lfloor=f_{S}^{0}\lfloor\right.
$$

We now turn to find relations between the kernels defined above and algebraic shifting.

\section{Shifting and kernels of boundary operations}

In this section we give some equivalent descriptions of the algebraic shifting operator, using the kernels defined in Section 2. This approach will be used throughout this work.

The following generalizes a result for graphs [3] (the proof is similar):

Proposition 3.1 Let $R \subseteq[n],|R|<j+1$. Then

$$
\operatorname{Ker}_{j} f_{R} L=\bigcap_{i:[n] \ni i \notin R} \operatorname{Ker}_{j} f_{R \cup i} L .
$$

Proof: Recall that $h\left\lfloor\left(g\lfloor f)=(h \wedge g)\left\lfloor f\right.\right.\right.$. Thus, if $f_{R}\lfloor m=0$ then

$$
f_{R \cup i}\left\lfloor m= \pm f_{i}\left\lfloor\left( f_{R}\lfloor m)=f_{i}\lfloor 0=0 .\right.\right.\right.
$$

Now suppose $m \in \operatorname{span}\left\{e_{S}: S \in K,|S|=j+1\right\} \backslash \operatorname{Ker}_{j} f_{R}\left\lfloor\right.$. The set $\left\{f_{Q}^{*}: Q \subseteq[n],|Q|=\right.$ $j+1-|R|\}$ forms a basis of $\left(\bigwedge^{j+1-|R|} V\right)^{*}$, so there is some $f_{R^{\prime}}, R^{\prime} \subseteq[n],\left|R^{\prime}\right|=j+1-|R|$, 
(note that $R^{\prime} \neq \emptyset$ ) such that

$$
<f_{R^{\prime}} \wedge f_{R}, m>=<f_{R^{\prime}}, f_{R}\lfloor m>\neq 0
$$

We get that for $i_{0} \in R^{\prime}: i_{0} \notin R$ and $<f_{R^{\prime} \backslash i_{0}}, f_{R \cup i_{0}}\left\lfloor m>\neq 0\right.$. Thus $m \notin \operatorname{Ker}_{j} f_{R \cup i_{0}}\lfloor$ which completes the proof.

In the next proposition we determine the shifting of a simplicial complex by looking at the intersection of kernels of boundary operations (actually only at their dimensions): Let $S$ be a subset of $[n]$ of size $s$. For $R \subseteq[n],|R|=s$, we look at $f_{R}\left\lfloor: \bigwedge^{s}(K) \rightarrow \bigwedge^{s-|R|}(K)=\mathbb{R}\right.$.

Proposition 3.2 Let $K_{0}, S \subseteq[n],\left|K_{0}\right|=k,|S|=s$. The following quantities are equal:

$$
\begin{gathered}
\operatorname{dim} \bigcap_{R<{ }_{L} S,|R|=s, R \subseteq[n]} \operatorname{Ker}_{s-1} f_{R}\lfloor, \\
\operatorname{dim} \bigcap_{\substack{R<L \\
S},|R|=s, R \subseteq[k]} \operatorname{Ker}_{s-1} f_{R}^{0}\lfloor, \\
\left|\left\{T \in \Delta(K):|T|=s, S \leq_{L} T\right\}\right| .
\end{gathered}
$$

In particular, $S \in \Delta(K)$ iff

$$
\operatorname{dim} \bigcap_{R<L} \bigcap_{L,|R|=s, R \subseteq[n]} \operatorname{Ker}_{s-1} f_{R} \mathrm{~L}>\operatorname{dim} \bigcap_{R \leq L} \bigcap_{S,|R|=s, R \subseteq[n]} \operatorname{Ker}_{s-1} f_{R} \mathrm{~L}
$$

(equivalently, $S \in \Delta(K)$ iff $\bigcap_{R<{ }_{L} S,|R|=s, R \subseteq[n]} \operatorname{Ker}_{s-1} f_{R}\left\llcorner\nsubseteq \operatorname{Ker}_{s-1} f_{S}\llcorner\right.$ ).

Proof: First we show that (5) equals (6). For every $T \subseteq[n], T<_{L} S$, decompose $T=$ $T_{1} \cup T_{2}$, where $T_{1} \subseteq[k], T_{2} \cap[k]=\emptyset$. For each $T_{3}$ satisfying $T_{3} \subseteq[k], T_{3} \supseteq T_{1},\left|T_{3}\right|=|T|$, we have $T_{3} \leq{ }_{L} T$. Each $f_{t}^{0}$, where $t \in T_{2}$, is a linear combination of the $f_{i}^{0}$ 's, $1 \leq i \leq k$, so $f_{T}^{0}$ is a linear combination of such $f_{T_{3}}^{0}$ 's. Thus, for every $j \geq s-1$,

$$
\bigcap_{R \leq L} \operatorname{Ker}_{j} f_{R}^{0}\left\llcorner\subseteq \operatorname{Ker}_{j} f_{T}^{0}\llcorner,\right.
$$

and hence

$$
\bigcap_{R<L} \operatorname{Ker}_{j} f_{R}^{0}\left\lfloor=\bigcap_{R<L]} \bigcap_{L} \operatorname{Ker}_{j} f_{R}^{0}\lfloor.\right.
$$

Combining with (4) the desired equality follows. 
Next we show that (7) equals (6). Let $m \in \bigwedge^{s}(K)$ and $R \subseteq[n],|R|=s$. Let us express $m$ and $f_{R}$ in the basis $\left\{e_{S}: S \subseteq[n]\right\}$ :

$$
\begin{aligned}
m & =\sum_{T \in K,|T|=s} \gamma_{T} e_{T} \\
f_{R} & =\sum_{S^{\prime} \subseteq[n],\left|S^{\prime}\right|=s} A_{R S^{\prime}} e_{S^{\prime}}
\end{aligned}
$$

where $A_{R S^{\prime}}$ is the minor of $A$ (transition matrix) with respect to the rows $R$ and columns $S^{\prime}$, and where $\gamma_{T}$ is a scalar in $\mathbb{R}$.

By bilinearity we get

$$
f_{R}^{0}\left\lfloor m=f_{R}\left\lfloor m=\sum_{T \in K,|T|=s} \gamma_{T} A_{R T}\right.\right.
$$

Thus (6) equals the dimension of the solution space of the system $B_{S} x=0$, where $B_{S}$ is the matrix $\left(A_{R T}\right)$, where $R<_{L} S, R \subseteq[k],|R|=s$ and $T \in K,|T|=s$. But, since the row indices of $B_{S}$ are an initial set with respect to the lexicographic order, the intersection of $\Delta(K)$ with this set of indices determines a basis of the row space of $B_{S}$. Thus, $\operatorname{rank}\left(B_{S}\right)=\left|\left\{R \in \Delta(K):|R|=s, R<_{L} S\right\}\right|$. But $K$ and $\Delta(K)$ have the same $f$-vector, so we get:

$$
\begin{gathered}
\operatorname{dim} \bigcap_{R<{ }_{L} S, R \subseteq[k],|R|=s} \operatorname{Ker}_{s-1} f_{R}^{0} L=f_{s-1}(K)-\operatorname{rank}\left(B_{S}\right) \\
=\left|\left\{T \in \Delta(K):|T|=s, S \leq_{L} T\right\}\right|
\end{gathered}
$$

as desired.

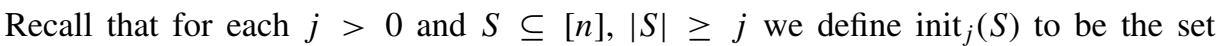
of lexicographically least $j$ elements in $S$, and for every $i>0 I_{S}^{i}=I_{S}^{i}(n)=\{T: T \subseteq$ $\left.[n],|T|=|S|+i, \operatorname{init}_{|S|}(T)=S\right\}$. Let $S_{(i)}^{(m)}=S_{(i)}^{(m)}(n)=\min _{<_{L}} I_{S}^{i}(n)$ and $S_{(i)}^{(M)}=S_{(i)}^{(M)}(n)=$ $\max _{<L} I_{S}^{i}(n)$. In the sequel, all the sets of numbers we consider are subsets of $[n]$. In order to simplify notation, we will often omit noting that. We get the following information about the partition of the faces in the shifted complex into 'intervals':

Proposition 3.3 Let $K_{0} \subseteq[n], S \subseteq[n], i>0$. Then

$$
\left|I_{S}^{i} \cap \Delta(K)\right|=\operatorname{dim} \bigcap_{R<L} \operatorname{Ker}_{|S|+i-1} f_{R} L(K)-\operatorname{dim} \bigcap_{R \leq L} \operatorname{Ker}_{|S|+i-1} f_{R} L(K) .
$$

Proof: By Proposition 3.1, 


$$
\begin{aligned}
& \operatorname{dim} \bigcap_{R<L} \operatorname{Ker}_{|S|+i-1} f_{R} L=\operatorname{dim} \bigcap_{R<{ }_{L} S} \bigcap_{j \notin R, j \in[n]} \operatorname{Ker}_{|S|+i-1} f_{R \cup j} L=\cdots \\
& \quad=\operatorname{dim} \bigcap_{R<L} \bigcap_{T: T \cap R=\emptyset,|T|=i} \operatorname{Ker}_{|S|+i-1} f_{R \cup T} L=\operatorname{dim} \bigcap_{R<L} \operatorname{Ker}_{S_{(i)}^{(m)}} \operatorname{Ker}_{\left|S_{(i)}^{(m)}\right|-1} f_{R} L .
\end{aligned}
$$

(To see that the last equation is true, one needs to check that $\{R \cup T: T \cap R=\emptyset,|T|=$ $\left.\left.i, R<_{L} S\right\}=\left\{Q: Q<_{L} S_{(i)}^{(m)}\right\}\right)$. By Proposition 3.2,

$$
\operatorname{dim} \bigcap_{R<L} \operatorname{Ker}_{(i)}^{(m)} \operatorname{Ker}_{\mid(i)}^{(m)}\left|-1=1, f_{R} L=\right|\left\{Q \in \Delta(K):|Q|=|S|+i, S_{(i)}^{(m)} \leq_{L} Q\right\} \mid .
$$

Similarly,

$$
\operatorname{dim} \bigcap_{R \leq L} \operatorname{Ker}_{|S|+i-1} f_{R} L(K)=\left|\left\{F \in \Delta(K):|F|=|S|+i, S_{(i)}^{(M)}<_{L} F\right\}\right| .
$$

(Here one checks that $\left\{R \cup T: T \cap R=\emptyset,|T|=i, R \leq_{L} S\right\}=\left\{F: F \leq_{L} S_{(i)}^{(M)}\right\}$ ). Thus, the proof of the proposition is completed.

Note that on $I_{S}^{1}$ the lexicographic order and the partial order $<_{P}$ coincide, since all sets in $I_{S}^{1}$ have the same $|S|$ least elements. As $\Delta(K)$ is shifted, $I_{S}^{1} \cap \Delta(K)$ is an initial set of $I_{S}^{1}$ with respect to $<_{L}$. Denote for short

$$
D(S)=D_{K}(S)=\left|I_{\text {init }|S|-1^{1}(S)}(n) \cap \Delta(K)\right| .
$$

$D_{K}(S)$ is indeed independent of the particular $n$ we choose, as long as $K_{0} \subseteq[n]$. We observe that

Proposition 3.4 Let $K_{0}$ and $S=\left\{s_{1}<\cdots<s_{j}<s_{j+1}\right\}$ be subsets of [ $\left.n\right]$. Then $S \in \Delta(K) \Leftrightarrow s_{j+1}-s_{j} \leq D(S)$.

Another easy preparatory lemma is the following:

Proposition 3.5 Let $K_{0}, S \subseteq[n]$. Then $D_{\Delta(K)}(S)=D_{K}(S)$.

Proof: It follows from the fact that $\Delta^{2}=\Delta$ (Kalai [4], or later on here in Corollary 5.7).

\section{Shifting union of simplicial complexes}

Let us consider a general union first:

Problem 4.1 ([6], Problem 13) Given two simplicial complexes $K$ and $L$, find all possible connections between $\Delta(K \cup L), \Delta(K), \Delta(L)$ and $\Delta(K \cap L)$. 
We look at $\bigwedge(K \cup L), \bigwedge(K \cap L), \bigwedge(K)$ and $\bigwedge(L)$ as subspaces of $\bigwedge(V)$ where $V=$ $\operatorname{span}\left\{e_{1}, \ldots, e_{n}\right\}$ and $[n]=(K \cup L)_{0}$. As before, the $f_{i}$ 's are generic linear combinations of the $e_{j}$ 's where $j \in[n]$. Let $S \subseteq[n],|S|=s$ and $1 \leq j$. First we find a connection between boundary operations on the spaces associated with $K, L, K \cap L$ and $K \cup L$ via the following commutative diagram of exact sequences:

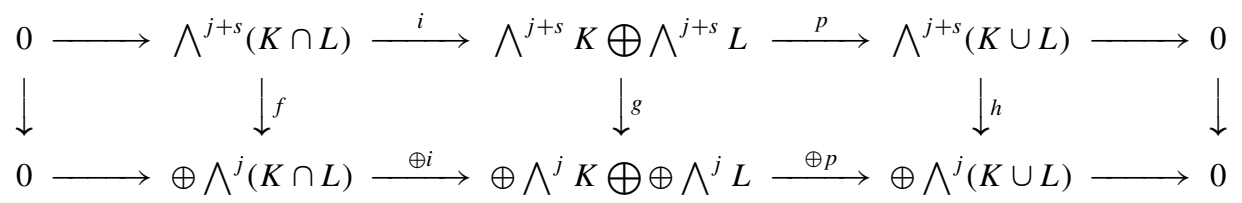

where all sums $\oplus$ in the bottom sequence are taken over $\left\{A: A<_{L} \quad S,|A|=s\right\}$ and $i(m)=(m,-m), p((a, b))=a+b, \oplus i(m)=(m,-m), \oplus p((a, b))=a+b, f=$ $\oplus_{A<{ }_{L} S} f_{A} \mathrm{~L}(K \cap L), g=\left(\oplus_{A<{ }_{L} S} f_{A} \mathrm{~L}(K), \oplus_{A<{ }_{L} S} f_{A} \mathrm{~L}(L)\right)$ and $h=\oplus_{A<{ }_{L} S} f_{A} \mathrm{~L}(K \cup L)$.

By the snake lemma, (8) gives rise to the following exact sequence:

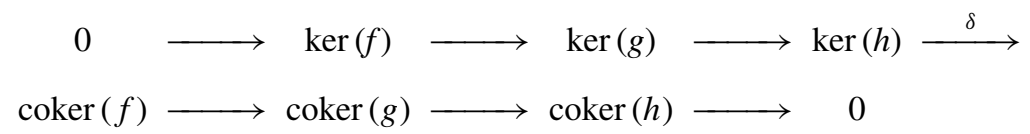

where $\delta$ is the connecting homomorphism. Let $\left(8^{\prime}\right)$ be the diagram obtained from (8) by replacing $A<_{L} S$ with $A \leq_{L} S$ everywhere, and renaming the maps by adding a superscript to each of them. Let $\left(9^{\prime}\right)$ be the sequence derived from $\left(8^{\prime}\right)$ by applying to it the snake lemma. If $\delta=0$ in (9), and also the connecting homomorphism $\delta^{\prime}=0$ in $\left(9^{\prime}\right)$, then by Proposition 3.3 the following additive formula holds:

$$
\left|I_{S}^{j} \cap \Delta(K \cup L)\right|=\left|I_{S}^{j} \cap \Delta(K)\right|+\left|I_{S}^{j} \cap \Delta(L)\right|-\left|I_{S}^{j} \cap \Delta(K \cap L)\right| .
$$

\subsection{A proof of Theorem 1.1}

Proof of Theorem 1.1: Put $j=d+2$ in (8) and in $\left(8^{\prime}\right)$. Thus, the range and domain of $f$ in $(8)$ and of $f^{\prime}$ in $\left(8^{\prime}\right)$ are zero, hence $\operatorname{ker} f=\operatorname{coker} f=0$ and $\operatorname{ker} f^{\prime}=\operatorname{coker} f^{\prime}=0$, and Theorem 1.1 follows.

Remark It would be interesting to understand what extra information about $\Delta(K \cup L)$ we can derive by using more of the structure of $\Delta(K \cap L)$, and not merely its dimension. In particular, it would be interesting to find combinatorial conditions that imply the vanishing of $\delta$ in (9). The proof of Theorem 1.2 in Section 4.4 provides a step in this direction. The 
Mayer-Vietoris long exact sequence (see [7] p. 186) gives some information of this type, by interpreting of the Betti vector using the shifted complex [1], mentioned in Section 2.

\subsection{How to shift a disjoint union?}

As a corollary to Theorem 1.1 we get the following combinatorial formula for shifting the disjoint union of simplicial complexes:

Theorem 4.2 Let $(K \dot{\cup} L)_{0}=[n],[n] \supseteq S=\left\{s_{1}<\cdots<s_{j}<s_{j+1}\right\}$. Then

$$
S \in \Delta(K \dot{\cup} L) \Leftrightarrow s_{j+1}-s_{j} \leq\left|I_{\text {init }_{|S|-1}(S)}^{1} \cap \Delta(K)\right|+\left|I_{\text {init }_{|S|-1}(S)}^{1} \cap \Delta(L)\right| .
$$

Proof: Put $d=-1$ and $A=\operatorname{init}_{|S|-1}(S)$ in Theorem 1.1, and by Proposition 3.4 we are done.

As a corollary, we get the following nice equation, proposed by Kalai [6]:

Corollary 4.3 $\Delta(K \dot{\cup} L)=\Delta(\Delta(K) \dot{\cup} \Delta(L))$.

Proof: $\quad S \in \Delta(K \cup L)$ iff (by Theorem 4.2) $s_{j+1}-s_{j} \leq D_{K}(S)+D_{L}(S)$ iff (by Proposition 3.5) $s_{j+1}-s_{j} \leq D_{\Delta(K)}(S)+D_{\Delta(L)}(S)$ iff (by Theorem 4.2) $S \in \Delta(\Delta(K) \dot{\cup} \Delta(L)$ ).

\section{Remarks}

(1) Beyond a high enough dimension (to be specified) all faces of the shifting of a union are determined by the shifting of its components. Let $\operatorname{st}(K \cap L)=\{\sigma \in K \cup L$ : $\left.\sigma \cap(K \cap L)_{0} \neq \emptyset\right\}$. Then $\Delta(K)$ and $\Delta(L)$ determine all faces of $\Delta(K \cup L)$ of dimension $>\operatorname{dim}(\operatorname{st}(K \cap L))$, by applying Theorem 4.2 to the subcomplex of $K \cup L$ spanned by the vertices $(K \cup L)_{0}-(K \cap L)_{0}$.

(2) Let $X$ be a $(k+l) \times(k+l)$ generic block matrix, with an upper block of size $k \times k$ and a lower block of size $l \times l$. Although we defined the shifting operator $\Delta=\Delta_{A}$ with respect to a generic matrix $A$, the definition makes sense for any nonsingular matrix (but in that case the resulting complex may not be shifted). Let $K_{0}=[k]$ and $L_{0}=[k+1, k+l]$. Corollary 4.3 can be formulated as

$$
\Delta \circ \Delta_{X}(K \dot{\cup} L)=\Delta(K \dot{\cup} L)
$$

because $\Delta_{X}(K \dot{\cup} L)=\Delta(K) \dot{\cup} \Delta(L)$ (on the right hand side of the equation the vertices of the two shifted complexes are considered as two disjoint sets). However, there are simplicial complexes $C$ on $k+l$ vertices, for which $\Delta \circ \Delta_{X}(C) \neq \Delta(C)$. For example, let $k=l=3$ and take the graph $G$ of the octahedron $\{\{1\},\{4\}\} *\{\{2\},\{5\}\} *\{\{3\},\{6\}\}$. Then $\Delta \circ \Delta_{X}(G) \ni\{4,5\} \notin \Delta(G)$. 
(3) By induction, we get from Corollary 4.3 that:

$$
\Delta\left(\dot{\cup}_{1 \leq i \leq n} K^{i}\right)=\Delta\left(\dot{\cup}_{1 \leq i \leq n} \Delta\left(K^{i}\right)\right)
$$

for any positive integer $n$ and disjoint simplicial complexes $K^{i}$.

(4) Theorem 4.2 gives a very simple (linear time in $t=2^{n}$ ) algorithm for computing $\Delta(K \cup \dot{\cup})$, given $\Delta(K)$ and $\Delta(L), n=\left|(K \cup L)_{0}\right|$.

(5) For symmetric algebraic shifting, introduced by Kalai [5], the results about shifting the disjoint union, namely the symmetric assertions analogous to Theorem 4.2 and Corollary 4.3, remain true. Techniques similar to those developed in Sections 3 and in the proof of Theorem 1.1 provide a proof of the symmetric analogue of Theorem 1.1 in the case where $d=-1$. We omit the details. For a general union, we do not know whether the symmetric analogue of Theorem 1.1 holds or not.

\subsection{A recursive formula for shifting a disjoint union}

We now turn to prove a recursive formula for computing $\Delta(K \dot{\cup} L)$, given $\Delta(K)$ and $\Delta(L)$, conjectured by Kalai [6].

We introduce the $\sqcup$ operator (as in [6]) defined on shifted simplicial complexes. Let $K, L$ be shifted simplicial complexes. Define $K \sqcup L$ to be the simplicial yet to be shown complex satisfying $(K \sqcup L)_{0}=K_{0} \dot{\cup} L_{0}=[k+l]$ (assuming $K_{0}=[k], L_{0}=[l]$, but regarding $K_{0}, L_{0}$ as disjoint sets) and recursively satisfying

$$
(K \sqcup L)_{j}=1 *(\operatorname{lk}(1, K) \sqcup \operatorname{lk}(1, L))_{j-1} \cup(\operatorname{ast}(1, K) \sqcup \operatorname{ast}(1, L))_{j}
$$

where the link and anti-star of $S \in K$ are defined by:

$$
\begin{aligned}
\operatorname{lk}(S, K) & =\{T \in K: T \cap S=\emptyset, T \cup S \in K\}, \\
\operatorname{ast}(S, K) & =\{T \in K: T \cap S=\emptyset\} .
\end{aligned}
$$

Note that $\operatorname{lk}(S, K) \subseteq \operatorname{ast}(S, K)$. The $1 *()$ operator on sets of sets is defined (unusually) as: $1 * S=\{1 \cup \dot{s} s: s \in S\}$ for $S$ such that for all $s \in S 1 \notin s$. Kalai conjectured:

Corollary 4.4 Let $K$, L be two shifted simplicial complex. Then

$$
\Delta(K \cup \dot{U} L)=K \sqcup L
$$

Proof: By definition $(K \sqcup L)_{0}=\Delta(K \cup \dot{\cup} L)_{0}$. We proceed by induction on $n=\left|(K \cup \dot{\cup} L)_{0}\right|$. By the induction hypothesis, for every $j$

$$
(K \sqcup L)_{j}=\left[1 * \Delta(\operatorname{lk}(1, K) \dot{\cup} \operatorname{lk}(1, L))_{j-1}\right] \cup\left[\Delta(\operatorname{ast}(1, K) \dot{\cup} \operatorname{ast}(1, L))_{j}\right] .
$$


Denoting

$$
\begin{aligned}
\text { Ast } & =\operatorname{ast}(1, K) \dot{\operatorname{ast}}(1, L), \\
L k & =\operatorname{lk}(1, K) \dot{\cup} \operatorname{lk}(1, L),
\end{aligned}
$$

we have

$$
K \sqcup L=1 * \Delta(L k) \cup \Delta(\text { Ast })
$$

First we show that $K \sqcup L$ is a simplicial complex: $L k \subseteq$ Ast are two simplicial complexes, therefore $\Delta(L k) \subseteq \Delta$ (Ast), and we get that $K \sqcup L$ is also a simplicial complex. Our second step is to show that $K \sqcup L$ is shifted. As $1 * \Delta(L k)$ (not a simplicial complex) and $\Delta$ (Ast) are shifted, we only have to show that

$$
\partial(\Delta(\mathrm{Ast})) \subseteq \Delta(L k)
$$

(For a set $A \subseteq\left(\begin{array}{c}{[n]} \\ k\end{array}\right), \partial(A)=\left\{b \in\left(\begin{array}{c}{[n]} \\ k-1\end{array}\right): \exists a \in A\right.$ such that $\left.b \subseteq a\right\}$. For a simplicial complex $K, \partial(K)=\bigcup_{i} \partial\left(K_{i}\right)$.) A basic property of algebraic shifting [6] is that for every simplicial complex $C$,

$$
\partial(\Delta(C)) \subseteq \Delta(\partial(C))
$$

so we get

$$
\partial(\Delta(\mathrm{Ast})) \subseteq \Delta(\partial(\mathrm{Ast}))
$$

As $K$ and $L$ are shifted, $\partial(\operatorname{ast}(1, K)) \subseteq 1 \mathrm{k}(1, K)$, and similarly for $L$. Hence $\partial($ Ast $) \subseteq L k$, and therefore $\Delta(\partial($ Ast $)) \subseteq \Delta(L k)$. Now that we know that $K \sqcup L$ is a shifted simplicial complex, by Proposition 3.4 it is enough to show that for each $S \subseteq[n]$, we have

$$
D_{K \sqcup L}(S)=D_{\Delta(K \cup \dot{\cup})}(S),
$$

as $(K \sqcup L) \cap I_{\text {init }_{|S|-1}(S)}^{1}$ is an initial set of $I_{\text {init }_{|S|-1}}^{1}(S)$.

Case 1: $1 \notin S$, For each $T \in I_{\text {init }_{|S|-1}}^{1}(S) .1 \notin T$, therefore

$$
\begin{aligned}
D_{K \sqcup L}(S) & =D_{\Delta(\text { Ast })}(S)=D_{\text {ast }(1, K)}(S)+D_{\text {ast }(1, L)}(S) \\
& =D_{K}(S)+D_{L}(S)=D_{\Delta(K \cup \dot{ } L)}(S) .
\end{aligned}
$$

The second and last equations are by Theorem 4.2 and Proposition 3.5.

Case 2: $1 \in S$, For each $T \in I_{\text {init }_{|S|-1}}^{1}(S) .1 \in T$ and moreover, if $S^{\prime}=S \backslash\{1\}$, then $I_{\text {init }_{|S|-1}}^{1}(S)=1 * I_{\text {init }_{\left|S^{\prime}\right|-1}}^{1}\left(S^{\prime}\right)$. Thus

$$
\begin{aligned}
D_{K \sqcup L}(S) & =D_{\Delta(L k)}\left(S^{\prime}\right)=D_{\mathrm{lk}(1, K)}\left(S^{\prime}\right)+D_{\operatorname{lk}(1, L)}\left(S^{\prime}\right) \\
& =D_{K}(S)+D_{L}(S)=D_{\Delta(K \cup \dot{L})}(S) .
\end{aligned}
$$


The second and last equations are by Theorem 4.2 and Proposition 3.5. This completes the proof.

\subsection{How to shift a union over a simplex?}

In the case where $K \cap L=<\sigma>$ is a simplex (and all of its subsets), we also get a formula for $\Delta(K \cup L)$ in terms of $\Delta(K), \Delta(L)$, and $\Delta(K \cap L)$. This case corresponds to the topological operation called connected sum.

Proof of Theorem 1.2: For a simplicial complex $H$, let $\bar{H}$ denote the complete simplicial complex $2^{H_{0}}$. The inclusions $H \hookrightarrow \bar{H}$ for $H=K, L,<\sigma>$ induce a morphism from the commutative diagram (8) of $K$ and $L$ to the analogous commutative diagram $(\overline{8})$ of $\bar{K}$ and $\bar{L}$. By functoriality of the sequence of the snake lemma, we obtain the following commutative diagram:

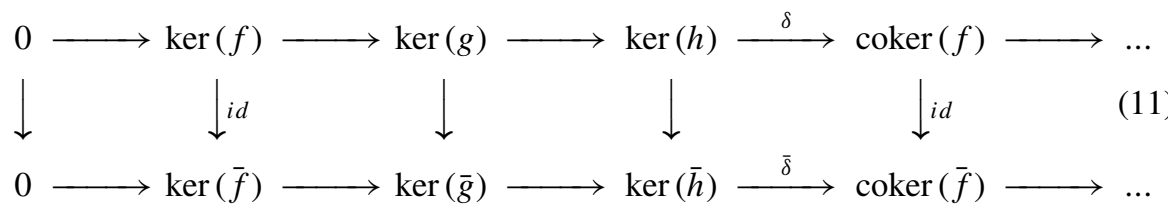

where the bars indicate that $(\overline{8})$ is obtained from (8) by putting bars over all the complexes and renaming the maps by adding a bar over each map. Thus, if $\bar{\delta}=0$ then also $\delta=0$, which, as we have seen, implies (10). The fact that $\Delta\left(2^{[|\sigma|]}\right)=\langle\sigma>$ completes the proof.

We show now that $\bar{\delta}=0$. To simplify notation, assume that $K$ and $L$ are complete complexes whose intersection is $\langle\sigma\rangle$ (which is a complete complex). Consider (8) with $j=1$. (It is enough to prove Theorem 1.2 for $i=1$ as for every $i>1, S \subseteq[n]$ and a simplicial complex $H$ on $[n], I_{S}^{i} \cap H=\biguplus_{T \in I_{S}^{i-1}}\left(I_{T}^{1} \cap H\right)$.) Let $m=m_{K}+m_{L} \in$ $\operatorname{ker}(h)$ where $\operatorname{supp}\left(m_{K}\right) \subseteq K \backslash<\sigma>$. By commutativity of the middle right square of (8), $\oplus_{A<{ }_{L} S} f_{A}\left\lfloor\left(m_{K}\right), \oplus_{A<{ }_{L} S} f_{A}\left\lfloor\left(m_{L}\right) \in \oplus_{A<{ }_{L} S} \bigwedge^{1}(\sigma)\right.\right.$. If we show that

$$
\oplus_{A<L} S f_{A} \mathrm{~L}\left(\bigwedge^{1+|S|} \sigma\right)=\oplus_{A<_{L} S} f_{A} \mathrm{~L}\left(\bigwedge^{1+|S|} K\right) \bigcap \oplus_{A{ }_{L} S} \bigwedge^{1}(\sigma),
$$

then there exists $m^{\prime} \in \bigwedge^{1+|S|}(\sigma)$ such that $\oplus_{A<_{L} S} f_{A}\left\lfloor\left(m_{K}\right)=\oplus_{A<_{L} S} f_{A}\left\lfloor\left(m^{\prime}\right)=f\left(m^{\prime}\right)\right.\right.$, hence $\delta(m)=\left[f\left(m^{\prime}\right)\right]=0$ (where $[c]$ denotes the image of $c$ under the projection onto coker $(f)$ ) i.e. $\delta=0$. (12) follows from the intrinsic characterization of the image of the maps it involves, given in Proposition 4.5. By Proposition 4.5, the right hand side of (12) consists of all $x \in \oplus_{R<_{L} S} \bigwedge^{1} K$ that satisfy $(a)$ and $(b)$ of Proposition 4.5 which are actually in $\oplus_{A<L} S \bigwedge^{1}(\sigma)$. By Proposition 4.5, this is exactly the left hand side of (12).

The following generalizes a result of Kalai for graphs ([3], Lemma 3.7). 
Proposition 4.5 Let $H$ be a complete simplicial complex with $H_{0} \subseteq[n]$, and let $S \subseteq[n]$, $|S|=s$. Then $\oplus_{R<_{L} S} f_{R} L\left(\bigwedge^{1+s} H\right)$ is the set of all $x=\left(x_{R}: R<_{L} S\right) \in \oplus_{R<_{L} S} \bigwedge^{1} H$ satisfying the following:

(a) For all pairs $(i, R)$ such that $i \in R<_{L} S,<f_{i}, x_{R}>=0$.

(b) For all pairs $(A, B)$ such that $A<_{L} S, B<_{L} S$, and $|A \Delta B|=2$, let $\{a\}=A \backslash B$ and $\{b\}=B \backslash A$. Then

$$
-<f_{b}, x_{A}>=(-1)^{s g n_{A \cup B}(a, b)}<f_{a}, x_{B}>
$$

where $\operatorname{sgn}_{A \cup B}(a, b)$ is the number modulo 2 of elements between $a$ and $b$ in the ordered set $A \cup B$.

Proof: Let us verify first that every element in $I m=\oplus_{R<{ }_{L} S} f_{R} L\left(\bigwedge^{1+s} H\right)$ satisfies (a) and $(b)$. Let $y \in \bigwedge^{1+s} H$. If $i \in R<{ }_{L} S$ then $<f_{i}, f_{R}\left\lfloor y>=<f_{i} \wedge f_{R}, y>=\right.$ $<0, y>=0$, hence $(a)$ holds. For $i \in T \subseteq[n]$ for some $n$, let $\operatorname{sgn}(i, T)=\mid\{t \in T$ : $t<i\} \mid(\bmod 2)$. If $A, B<_{L} S,\{a\}=A \backslash B$ and $\{b\}=B \backslash A$ then $-<f_{b}, f_{A}\left\lfloor y>=-<f_{b} \wedge\right.$ $f_{A}, y>=-(-1)^{\operatorname{sgn}(b, A \cup B)}<f_{A \cup B}, y>=-(-1)^{\operatorname{sgn}(b, A \cup B)}(-1)^{\operatorname{sgn}(a, A \cup B)}<f_{a} \wedge f_{B}, y>=$ $(-1)^{s g n_{A \cup B}(a, b)}<f_{a}, f_{B}\lfloor y>$, hence $(b)$ holds.

We showed that every element of $\operatorname{Im}$ satisfies $(a)$ and $(b)$. Denote by $X$ the space of all $x \in \oplus_{R<_{L} S} \wedge^{1} H$ satisfying $(a)$ and $(b)$. It remains to show that $\operatorname{dim}(X)=$ $\operatorname{dim}(\operatorname{Im})$.

Following the proof of Proposition 3.3, $\operatorname{dim}(\operatorname{Im})=\operatorname{dim}\left(\bigwedge^{1+s} H\right)-$ $\operatorname{dim}\left(\bigcap_{R<L} S \operatorname{Ker}_{s} f_{R}\left\lfloor\left(\bigwedge^{1+s} H\right)=|\{T \in \Delta(H):|T|=s+1\}|-\mid\{T \in \Delta(H):|T|=\right.\right.$ $\left.s+1, S_{(1)}^{(m)} \leq_{L} T\right\}|=|\left\{T \in \Delta(H):|T|=s+1\right.$, init $\left._{s}(T)<_{L} S\right\} \mid$. Let $h=\left|H_{0}\right|$ and $\operatorname{sum}(T)=\left|\left\{t \in T: T \backslash\{t\}<_{L} S\right\}\right|$. Note that $\Delta(H)=2^{[h]}$. Counting according to the initial $s$-sets, we conclude that in case $s<h$,

$$
\begin{aligned}
\operatorname{dim}(\operatorname{Im})= & \left|\left\{R: R<_{L} S, R \subseteq[h]\right\}\right|(h-s)-\sum\{\operatorname{sum}(T)-1: T \subseteq[h], \\
& \left.|T|=s+1, \operatorname{init}_{s}(T)<_{L} S, \operatorname{sum}(T)>1\right\} .
\end{aligned}
$$

In case $s \geq h, \operatorname{dim}(\operatorname{Im})=0$.

Now we calculate $\operatorname{dim}(X)$. Let us observe that every $x \in X$ is uniquely determined by its coordinates $x_{R}$ such that $R \subseteq[h]$. Let $i \in R \backslash[h], R<_{L} S$. Every $j \in[h] \backslash R$ gives rise to an equation $(b)$ for the pair $(R \cup j \backslash i, R)$ and every $j \in R \cap[h]$ gives rise to an equation $(a)$ for the pair $(j, R)$. Recall that $x_{R}$ is a linear combination of the form $x_{R}=\sum_{l \in[h]} \gamma_{l, R} e_{l}$ with scalars $\gamma_{l, R}$. Thus, we have a system of $h$ equations on the $h$ variables $\left(\gamma_{l, R}\right)_{l \in[h]}$ of $x_{R}$, with coefficients depending only on $x_{F}$ 's with $F<_{L} R$ (actually also $|F \cap[h]|=1+|R \cap[h]|$ ) and on the generic $f_{k}$ 's, $k \in[n]$. This system has a unique solution as the $f_{k}$ 's are generic. By repeating this argument we conclude that $x_{R}$ is determined by the coordinates $x_{F}$ such that $F \subseteq[h]$.

Let $x(h)$ be the restriction of $x \in X$ to its $\left\{x_{R}: R \subseteq[h], R<_{L} S\right\}$ coordinates, and let $X(h)=\{x(h): x \in X\}$. Then $\operatorname{dim}(X(h))=\operatorname{dim}(X)$.

Let $[a],[b]$ be the matrices corresponding to the equation systems $(a),(b)$ with variables $\left(\gamma_{l, T}\right)_{l \in[h], T{ }_{L} S}$ restricted to the cases $T \subseteq[h]$ and $A, B \subseteq[h]$, respectively. $[a]$ is an 
$s \cdot\left|\left\{R \subseteq[h]: R<_{L} S\right\}\right| \times h \cdot\left|\left\{R \subseteq[h]: R<_{L} S\right\}\right|$ matrix and $[b]$ is a $\mid\{A, B \subseteq[h]:$ $\left.A, B<_{L} S,|A \Delta B|=2\right\}|\times h \cdot|\left\{R \subseteq[h]: R<_{L} S\right\} \mid$ matrix.

We observe that the row spaces of $[a]$ and $[b]$ have a zero intersection. Indeed, for a fixed $R \subseteq[h]$, the row space of the restriction of $[a]$ to the $h$ columns of $R$ is $\operatorname{span}\left\{f_{i}^{0}: i \in R\right\}$ (recall that $f_{i}^{0}$ is the obvious projection of $f_{i}$ on the coordinates $\left\{e_{j}: j \in H_{0}\right\}$ ), and the row space of the restriction of $[b]$ to the $h$ columns of $R$ is $\operatorname{span}\left\{f_{j}^{0}: j \in[h] \backslash R\right\}$. But as the $f_{k}^{0}$ 's, $k \in[h]$, are generic, $\operatorname{span}\left\{f_{k}^{0}: k \in[h]\right\}=\bigwedge^{1} H$. Hence $\operatorname{span}\left\{f_{i}^{0}: i \in R\right\} \cap \operatorname{span}\left\{f_{j}^{0}: j \in\right.$ $[h] \backslash R\}=\{0\}$. We conclude that the row spaces of $[a]$ and $[b]$ have a zero intersection.

$[a]$ is a diagonal block matrix whose blocks are generic of size $s \times h$, hence

$$
\operatorname{rank}([a])=s \cdot\left|\left\{R: R<_{L} S, R \subseteq[h]\right\}\right|
$$

in case $s<h$.

Now we compute $\operatorname{rank}([b])$. For $T \subseteq[h],|T|=s+1$, let us consider the pairs in $(b)$ whose union is $T$. If $(A, B)$ and $(C, D)$ are such pairs, and $A \neq C$, then $(A, C)$ is also such a pair. In addition, if $A, B, C$ are different (the union of each two of them is $T$ ) then the three rows in $[b]$ indexed by $(A, B),(A, C)$ and $(B, C)$ are dependent; the difference between the first two equals the third. Thus, the row space of all pairs $(A, B)$ with $A \cup B=T$ is spanned by the rows indexed $\left(\operatorname{init}_{s}(T), B\right)$ where $\operatorname{init}_{s}(T) \cup B=T$.

We verify now that the rows $\bigcup\left\{\operatorname{init}_{s}(T), B\right): \operatorname{init}_{s}(T) \cup B=T \subseteq[h],|T|=s+$ $1,|B|=s\}$ of $[b]$ are independent. Suppose that we have a nontrivial linear dependence among these rows. Let $B^{\prime}$ be the lexicographically maximal element in the set of all $B$ 's appearing in the rows $(A, B)$ with nonzero coefficient in that dependence. There are at most $h-s$ rows with nonzero coefficient whose restriction to their $h$ columns of $B^{\prime}$ is nonzero (they correspond to $A$ 's with $A=\operatorname{init}_{s}(B \cup\{i\}$ where $i \in[h] \backslash B)$. Again, as the $f_{i}$ 's are generic, this means that the restriction of the linear dependence to the $h$ columns of $B^{\prime}$ is nonzero, a contradiction. Thus,

$$
\begin{aligned}
\operatorname{rank}([b]) & =\left|\bigcup_{B<L}\left\{\left(\operatorname{init}_{s}(T), B\right): \operatorname{init}_{s}(T) \cup B=T \subseteq[h],|T|=s+1,|B|=s\right\}\right| \\
& =\sum\left\{\operatorname{sum}(T)-1: T \subseteq[h],|T|=s+1, \operatorname{init}_{s}(T)<_{L} S, \operatorname{sum}(T)>1\right\} .
\end{aligned}
$$

(Note that indeed $B<_{L} S$ implies $\operatorname{init}_{S}(T)<_{L} S$ as $B \subseteq T$.)

For $s<h, \operatorname{dim}(X(h))=h \cdot\left|\left\{R: R<_{L} S, R \subseteq[h]\right\}\right|-\operatorname{rank}([a])-\operatorname{rank}([b])$, which by (13), (14) and (15) equals $\operatorname{dim}(\operatorname{Im})$. For $s \geq h, \operatorname{dim}(X(h))=0=\operatorname{dim}(\operatorname{Im})$. This completes the proof.

As a corollary of Theorem 1.2, we get the following combinatorial formula for shifting the union over a simplex of simplicial complexes:

Theorem 4.6 Let $K$ and $L$ be simplicial complexes where $K \cap L=<\sigma>$ is a complete 
simplicial complex. Let $(K \cup L)_{0}=[n],[n] \supseteq T=\left\{t_{1}<\cdots<t_{j}<t_{j+1}\right\}$. Then

$$
T \in \Delta(K \cup L) \Leftrightarrow t_{j+1}-t_{j} \leq D_{K}(T)+D_{L}(T)-D_{<\sigma>}(T) .
$$

In particular, any gluing of $K$ and L along a d-simplex results in the same shifted complex $\Delta(K \cup L)$, depending only on $\Delta(K), \Delta(L)$ and $d$.

Proof: Put $i=1$ and $S=\operatorname{init}_{|T|-1}(T)$ in Theorem 1.2, and by Proposition 3.4 we are done.

Remark For symmetric shifting, the assertions analogous to Theorems 1.2 and 4.6 remain true. For their proof one uses a symmetric variant of Proposition 4.5 (where condition $(a)$ is omitted, and condition (b) has a symmetric analogue).

\section{Shifting near cones}

A simplicial complex $K$ is called a near cone with respect to a vertex $v$ if for every $j \in S \in K$ also $v \cup S \backslash j \in K$. We are about to prove a decomposition theorem for the shifted complex of a near cone, from which the formula for shifting a cone (mentioned in the introduction) will follow. As a preparatory step we introduce the Sarkaria map, modified for homology.

\subsection{The Sarkaria map}

Let $K$ be a near cone with respect to a vertex $v=1$. Let $e=\sum_{i \in K_{0}} e_{i}$ and let $f=\sum_{i \in K_{0}} \alpha_{i} e_{i}$ be a linear combination of the $e_{i}$ 's such that $\alpha_{i} \neq 0$ for every $i \in K_{0}$. Imitating the Sarkaria maps for cohomology [8], we get for homology the following linear maps:

$$
\left(\bigwedge K, e_{v} \mathrm{~L}\right) \stackrel{U}{\longrightarrow}(\bigwedge K, e \mathrm{~L}) \stackrel{D}{\longrightarrow}(\bigwedge K, f \mathrm{~L})
$$

defined as follows: for $S \in K$

$$
\begin{aligned}
U\left(e_{S}\right) & = \begin{cases}e_{S}-\sum_{i \in S}(-1)^{s g n(i, S)} e_{v \cup S \backslash i} & \text { if } v \notin S \\
e_{S} & \text { if } v \in S\end{cases} \\
D^{-1}\left(e_{S}\right) & =\left(\prod_{i \in S} \alpha_{i}\right) e_{S} .
\end{aligned}
$$

It is justified to write $D^{-1}$ as all the $\alpha_{i}$ 's are non zero.

Proposition 5.1 The maps $U$ and $D$ are isomorphisms of chain complexes. In addition they satisfy the following 'grading preserving' property: if $S \cup T \in K, S \cap T=\emptyset$, then

$$
U\left(e_{S} \wedge e_{T}\right)=U\left(e_{S}\right) \wedge U\left(e_{T}\right) \text { and } \quad D\left(e_{S} \wedge e_{T}\right)=D\left(e_{S}\right) \wedge D\left(e_{T}\right)
$$


Proof: The check is straightforward. First we check that $U$ and $D$ are chain maps. Let $\alpha_{S}=\prod_{i \in S} \alpha_{i}$. For every $e_{S}$, where $S \in K, D$ satisfies

$$
D \circ e\left\lfloor\left(e_{S}\right)=D\left(\sum_{j \in S}(-1)^{s g n(j, S)} e_{S \backslash j}\right)=\sum_{j \in S}(-1)^{\operatorname{sgn}(j, S)} \frac{\alpha_{j}}{\alpha_{S}} e_{S \backslash j}\right.
$$

and

$$
f\left\lfloor\circ D\left(e_{S}\right)=f\left\lfloor\left(\frac{1}{\alpha_{S}} e_{S}\right)=\sum_{j \in S}(-1)^{s g n(j, S)} \frac{\alpha_{j}}{\alpha_{S}} e_{S \backslash j} .\right.\right.
$$

For $U$ : if $v \in S$ we have

$$
U \circ e_{v}\left\lfloor\left(e_{S}\right)=U\left(e_{S \backslash v}\right)=e_{S \backslash v}-\sum_{i \in S \backslash v}(-1)^{\operatorname{sgn}(i, S \backslash v)} e_{S \backslash i}=\sum_{j \in S}(-1)^{\operatorname{sgn}(j, S)} e_{S \backslash j}\right.
$$

The last equation holds because $v=1$. Further,

$$
e\left\lfloor\circ U\left(e_{S}\right)=e\left\lfloor\left(e_{S}\right)=\sum_{j \in S}(-1)^{s g n(j, S)} e_{S \backslash j}\right.\right.
$$

If $v \notin S$ we have

$$
U \circ e_{v} L\left(e_{S}\right)=U(0)=0
$$

and

$$
\begin{aligned}
e\left\lfloor\circ U\left(e_{S}\right)=\right. & e\left\lfloor\left(e_{S}\right)-e\left\lfloor\left(\sum_{j \in S}(-1)^{s g n(j, S)} e_{S \cup v \backslash j}\right)\right.\right. \\
= & \sum_{j \in S}(-1)^{s g n(j, S)} e_{S \backslash j}-\sum_{i \in S}(-1)^{s g n(i, S)} \sum_{t \in S \cup v \backslash i}(-1)^{s g n(t, S \cup v \backslash i)} e_{S \cup v \backslash\{i, t\}} \\
= & \sum_{j \in S}(-1)^{\operatorname{sgn}(j, S)} e_{S \backslash j}\left(1-(-1)^{\operatorname{sgn}(v, S \cup v \backslash j)}\right) \\
& -\sum_{j, i \in S, i \neq j}(-1)^{s g n(i, S)}(-1)^{s g n(j, S \cup v \backslash i)} e_{S \cup v \backslash\{i, j\}} .
\end{aligned}
$$

In the last line, the left sum is zero as $v=1$, and for the same reason the right sum can be written as:

$$
\sum_{j, i \in S, i<j}\left((-1)^{\operatorname{sgn}(i, S)+\operatorname{sgn}(j, S \backslash i)}+(-1)^{\operatorname{sgn}(j, S)+\operatorname{sgn}(i, S \backslash j)}\right) e_{S \cup v \backslash\{i, j\}} .
$$

As $i<j$, the $\{i j\}$ coefficient equals

$$
(-1)^{\operatorname{sgn}(i, S)+\operatorname{sgn}(j, S)+1}+(-1)^{\operatorname{sgn}(j, S)+\operatorname{sgn}(i, S)}=0,
$$


hence $e\left\llcorner\circ U\left(e_{S}\right)=U \circ e_{v}\left\llcorner\left(e_{S}\right)\right.\right.$ for every $S \in K$. By linearity of $U$ and $D$ (and of the boundary maps), we have that $U, D$ are chain maps. To show that $U, D$ are onto, it is enough to show that each $e_{S}$, where $S \in K$, is in their image. This is obvious for $D$. For $U$ : if $v \in S$ then $U\left(e_{S}\right)=e_{S}$, otherwise $e_{S}=U\left(e_{S}\right)+\sum_{i \in S}(-1)^{\operatorname{sgn}(i, S)} e_{v \cup S \backslash i}$, which is a linear combination of elements in $\operatorname{Im}(U)$, so $e_{S} \in \operatorname{Im}(U)$ as well. Comparing dimensions, $U$ and $D$ are also $1-1$.

We now show that $U$ 'preserves grading' in the described above sense (for $D$ it is clear). For disjoint subsets of $[n]$ define $\operatorname{sgn}(S, T)=|\{(s, t) \in S \times T: t<s\}|(\bmod 2)$. Let $S, T$ be disjoint sets such that $S \cup T \in K$. By $S \cup T$ we mean the ordered union of $S$ and $T$ (and similarly for other set unions).

Case 1: $v \notin S \cup T$.

$$
\begin{aligned}
U\left(e_{S}\right) \wedge U\left(e_{T}\right) & =e_{S} \wedge e_{T}+\sum_{i \in S}(-1)^{s g n(i, S)} e_{S \cup v \backslash i} \wedge e_{T}+\sum_{j \in T}(-1)^{\operatorname{sgn}(j, T)} e_{S} \wedge e_{T \cup v \backslash j} \\
& =(-1)^{\operatorname{sgn}(S, T)}\left(e_{S \cup T}+\sum_{l \in S \cup T}(-1)^{\operatorname{sgn}(l, S \cup T)} e_{S \cup T \cup v \backslash l}\right)=U\left(e_{S} \wedge e_{T}\right),
\end{aligned}
$$

where the middle equation uses the fact that $v=1$, which leads to the following sign calculation:

$$
\begin{aligned}
(-1)^{\operatorname{sgn}(i, S)}(-1)^{\operatorname{sgn}(S \cup v \backslash i, T)} & =(-1)^{\operatorname{sgn}(i, S)+\operatorname{sgn}(S \backslash i, T)}=(-1)^{\operatorname{sgn}(i, S)+\operatorname{sgn}(S, T)+\operatorname{sgn}(i, T)} \\
& =(-1)^{\operatorname{sgn}(S, T)}(-1)^{\operatorname{sgn}(i, S \cup T)} .
\end{aligned}
$$

Case 2: $v \in S \backslash T$.

$$
U\left(e_{S}\right) \wedge U\left(e_{T}\right)=e_{S} \wedge\left(e_{T}-\sum_{t \in T}(-1)^{\operatorname{sgn}(t, T)} e_{T \cup v \backslash t}\right)=e_{S} \wedge e_{T}=U\left(e_{S} \wedge e_{T}\right)
$$

Case 3: $v \in T \backslash S$. A similar calculation to the one for case 2 holds.

Remark The 'grading preserving' property of $U$ and $D$ extends to the case where $S \cap T \neq$ $\emptyset(S, T \in K)$, but we won't use it here. One has to check that in this case (where clearly $\left.e_{S} \wedge e_{T}=0\right)$ :

$$
U\left(e_{S}\right) \wedge U\left(e_{T}\right)=D\left(e_{S}\right) \wedge D\left(e_{T}\right)=0
$$

\subsection{Shifting a near cone}

We now prove a decomposition theorem for the shifted complex of a near cone.

Theorem 5.2 Let $K$ be a near cone on a vertex set $[n]$ with respect to a vertex $v=1$. Let $X=\left\{f_{i}: 1 \leq i \leq n\right\}$ be some basis of $\bigwedge^{1} K$ such that $f_{1}$ has no zero coefficients 
as a linear combination of some given basis elements $e_{i}$ 's of $\bigwedge^{1} K$, and such that for $g_{i-1}=f_{i}-<f_{i}, e_{1}>e_{1}, Y=\left\{g_{i}: 1 \leq i \leq n-1\right\}$ is a linearly independent set. Then

$$
\Delta_{X}(K)=\left(1 * \Delta_{Y}(\operatorname{lk}(v, K))\right) \cup B
$$

where $B$ is the set $\left\{S \in \Delta_{X}(K): 1 \notin S\right\}$.

Note that $1 * \Delta_{Y}(\mathrm{lk}(v, K))$ is not a simplicial complex, but merely a collection of faces of $\Delta_{X}(K)$ which contain 1 . We claim that this collection equals the set of all faces of $\Delta_{X}(K)$ which contain 1.

Proof: Clearly for every $l \geq 0$ : $\operatorname{Ker}_{l} e_{v} L=\bigwedge^{l+1}$ ast $(v, K)$ and $\operatorname{Im}_{l} e_{v} L=\bigwedge^{l} \operatorname{lk}(v, K)$. Using the Sarkaria map $D \circ U$ (see Proposition 5.1), we get that $I m f_{1} L$ is isomorphic to $\bigwedge \mathrm{k}(v, K)$ and is contained (because of 'grading preserving') in a sub-exterior-algebra generated by the elements $b_{i}=D U\left(e_{i}\right)=\frac{1}{\alpha_{i}} e_{i}-\frac{1}{\alpha_{v}} e_{v}, i \in K_{0} \backslash v$. Let $S \subseteq[n],|S|=$ $l, 1 \notin S$. Recall that $(g \wedge f)\lfloor h=g\lfloor(f\lfloor h)$. Now we are prepared to shift.

$$
\bigcap_{R<L} 1 \cup S \operatorname{Ker}_{l} f_{R} L \cong \operatorname{Ker}_{l} f_{1}\left\llcorner\oplus \bigcap _ { 1 \notin R < L } \operatorname { K e r } f _ { R } L \left(\operatorname{Im}_{l} f_{1}\llcorner\rightarrow \mathbb{R}),\right.\right.
$$

which by the Sarkaria map is isomorphic to

$$
\wedge^{l+1} \operatorname{ast}(v, K) \oplus \bigcap_{1 \notin T<L} \operatorname{Ker}\left((D U)^{-1} f_{T}\right)\left\lfloor\left(\wedge^{l} \operatorname{lk}(v, K) \rightarrow \mathbb{R}\right) .\right.
$$

Denote by $\pi_{t}$ the natural projection $\pi_{t}: \operatorname{span}\left\{e_{R}:|R|=t\right\} \rightarrow \operatorname{span}\left\{e_{R}:|R|=t, v \notin\right.$ $R\}$. Then

$$
\operatorname{Ker}\left((D U)^{-1} f_{T}\right)\left\lfloor\left(\wedge^{l} \operatorname{lk}(v, K) \rightarrow \mathbb{R}\right)=\operatorname{Ker}\left(\pi_{l}(D U)^{-1} f_{T}\right)\left\lfloor\left(\wedge^{l} \operatorname{lk}(v, K) \rightarrow \mathbb{R}\right)\right.\right.
$$

Now look at the matrix $\left(<\pi_{l} \circ(D U)^{-1} f_{T}, e_{R}>\right)$ where $1 \notin T<_{L} S, R \in \operatorname{lk}(v, K)_{l-1}$. It is obtained from the matrix $\left(<g_{T-1}, e_{R}>\right)$, where $1 \notin T<_{L} S, R \in \operatorname{lk}(v, K)_{l-1}$, by multiplying each column $R$ by the non-zero scalar $\prod_{i \in R} \alpha_{i}$ (by $T+i$ we mean the set $\{t+i: t \in T\}$ ). Thus, restricting to the first $m$ rows of each of these two matrices we get matrices of equal rank, for every $m$. This means, in terms of kernels and using the proof of Proposition 3.2, that in (16) we can replace $(D U)^{-1} f_{T}$ by $g_{T-1}$ (note that the proof of Proposition 3.2 can be applied to non-generic shifting as well). We get (putting $Q=T-1$ ):

$$
\operatorname{dim} \bigcap_{R<L} \operatorname{Ker}_{l} f_{R} L(K)=\operatorname{dim} \wedge^{l+1} \operatorname{ast}(v, K)+\operatorname{dim} \bigcap_{Q<L} \operatorname{Ker}_{l-1} g_{Q} L(\operatorname{lk}(v, K)) .
$$


As the left summand in the right hand side is a constant independent of $S$, it is canceled when applying the last part of Proposition 3.2, and we get:

$$
\begin{aligned}
& 1 \cup \dot{U} S \in \Delta_{X}(K) \Leftrightarrow \operatorname{dim} \bigcap_{R<{ }_{L} 1 \cup S} \operatorname{Ker}_{l} f_{R} L(K)>\operatorname{dim} \bigcap_{R \leq_{L} 1 \cup S} \operatorname{Ker}_{l} f_{R} L(K) \Leftrightarrow \\
& \quad \operatorname{dim} \bigcap_{T<<_{L} S-1} \operatorname{Ker}_{l-1} g_{T} L(\operatorname{lk}(v, K))>\operatorname{dim} \bigcap_{T \leq_{L} S-1} \operatorname{Ker}_{l-1} g_{T} L(\operatorname{lk}(v, K)) \Leftrightarrow \\
& \quad S-1 \in \Delta_{Y}(\operatorname{lk}(v, K)) .
\end{aligned}
$$

Thus we get the claimed decomposition of $\Delta_{X}(K)$.

As a corollary we get the following decomposition theorem for the generic shifted complex of a near cone.

Theorem 5.3 Let $K$ be a near cone with respect to a vertex $v$. Then

$$
\Delta(K)=(1 * \Delta(\operatorname{lk}(v, K))) \cup B,
$$

where $B$ is the set $\{S \in \Delta(K): 1 \notin S\}$.

Proof: Apply Theorem 5.2 for the case where $X$ is generic. In this case, $Y$ is also generic, and the theorem follows .

As a corollary we get the following property [6]:

Corollary $5.4 \Delta \circ$ Cone $=$ Cone $\circ \Delta$.

Proof: Consider a cone over $v$ : $\{v\} * K$. By Theorem 5.3, $\{1\} * \Delta(K) \subseteq \Delta(\{v\} * K)$, but those two simplicial complexes have equal $f$-vectors, and hence, $\{1\} * \Delta(K)=\Delta(\{v\} * K)$.

\section{Remarks}

(1) Note that by associativity of the join operation, we get by Corollary 5.4: $\Delta(K[m] * K)=$ $K[m] * \Delta(K)$ for every $m$, where $K[m]$ is the complete simplicial complex on $m$ vertices.

(2) Using the notation in Theorem 5.2 we get:

$$
\Delta_{X} \circ \text { Cone }=\text { Cone } \circ \Delta_{Y}
$$

Definition 5.5 $K$ is an $i$-near cone if there exist a sequence of simplicial complexes $K=K(0) \supset K(1) \supset \cdots \supset K(i)$ such that for every $1 \leq j \leq i$ there is a vertex $v_{j} \in K(j-1)$ such that $K(j)=\operatorname{ast}\left(v_{j}, K(j-1)\right)$ and $K(j-1)$ is a near cone w.r.t. $v_{j}$. 
Remark An equivalent formulation is that there exists a permutation $\pi: K_{0}=[n] \rightarrow[n]$ such that

$$
\pi(i) \in S \in K, 1 \leq l<i \Rightarrow(S \cup \pi(l) \backslash \pi(i)) \in K,
$$

which is more compact but less convenient for the proof of the following generalization of Theorem 5.3:

Corollary 5.6 Let $K$ be an i-near cone. Then

$$
\Delta(K)=B \cup \bigcup_{1 \leq j \leq i} j *\left(\Delta\left(\operatorname{lk}\left(v_{j}, K(j-1)\right)\right)+j\right),
$$

where $B=\{S \in \Delta(K): S \cap[i]=\emptyset\}$.

Proof: The case $i=1$ is Theorem 5.3.

By induction hypothesis, $\Delta(K)=\tilde{B} \cup \bigcup_{1 \leq j \leq i-1} j *(\Delta(K(j-1))+j)$ where $\tilde{B}=\{S \in$ $\Delta(K): S \cap[i-1]=\emptyset\}$. We have to show that

$$
\{S \in \Delta(K): \min \{j \in S\}=i\}=i *\left(\Delta\left(\operatorname{lk}\left(v_{i}, K(i-1)\right)\right)+i\right)
$$

For $|S|=l$ with $\min \{j \in S\}=i$, we have

$$
\bigcap_{R<L} \operatorname{Ker}_{l-1} f_{R} \mathrm{~L}(K)=\left(\bigcap_{j<i} \bigcap_{R:|R|=l, j \in R} \operatorname{Ker}_{l-1} f_{R} \mathrm{~L}\right) \cap\left(\bigcap_{R<L} \bigcap_{S: \min (R)=i} \operatorname{Ker}_{l-1} f_{R} \mathrm{~L}\right) .
$$

By repeated application of Proposition 3.1, for each $j<i$,

$$
\bigcap_{R:|R|=l, j \in R} \operatorname{Ker}_{l-1} f_{R} L=\operatorname{Ker}_{l-1} f_{j}
$$

Hence, (18) equals

$$
\left(\bigcap_{j<i} \operatorname{Ker}_{l-1} f_{j} \mathrm{~L}\right) \cap\left(\bigcap_{R<{ }_{L} S: \min (R)=i} \operatorname{Ker}_{l-1} f_{R} \mathrm{~L}\right)=\bigcap_{R<L} \bigcap_{S: \min (R)=i} \operatorname{Ker}_{l-1} f_{R} \mathrm{~L}(A),
$$

where $A=\bigcap_{j<i} \operatorname{Ker} f_{j} \mathrm{~L}(\bigwedge K)$, taking the kernels in each dimension.

By repeated application of the Sarkaria map, we get that $A \cong \bigwedge K(i-1)$ as 'graded' chain complexes. We will show now that

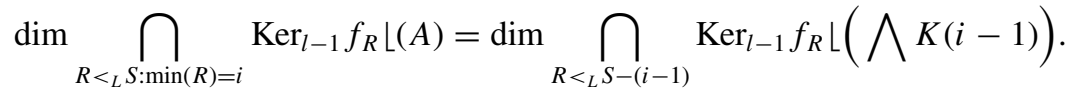


Let $\varphi: \bigwedge K(i-1) \rightarrow A$ be the Sarkaria isomorphism, and let $f$ be generic w.r.t. the basis $\left\{e_{i}, . ., e_{n}\right\}$ of $\bigwedge^{0} K(i-1)$. Then $\varphi(f)$ is generic w.r.t. the basis $\left\{\varphi\left(e_{i}\right), \ldots, \varphi\left(e_{n}\right)\right\}$ of $A$. We can choose a generic $\bar{f}$ w.r.t. $\left\{e_{1}, \ldots, e_{n}\right\}$ such that $<\bar{f}, \varphi\left(e_{j}\right)>=<\varphi(f), \varphi\left(e_{j}\right)>$ for every $i \leq j \leq n$. Actually, we can do so for $n-i$ generic $f_{j}$ 's simultaneously (as multiplying a nonsingular matrix over a field by a generic matrix over the same field results in a generic matrix over that field). We get that

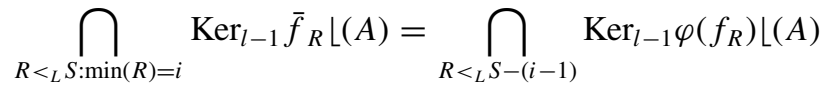

$$
\begin{aligned}
& \cong \bigcap_{R<L S-(i-1)} \operatorname{Ker}_{l-1} f_{R} L(\bigwedge K(i-1)) \text {. }
\end{aligned}
$$

As both the $f_{i}$ 's and the $\bar{f}_{i}$ 's are generic, $\bigcap_{R<L} S: \min (R)=i \operatorname{Ker}_{l-1} f_{R} L(A) \cong \bigcap_{R<L} S: \min (R)=i$ $\operatorname{Ker}_{l-1} \bar{f}_{R} L(A)$ and (19) follows. By applying Theorem 5.3 to the near cone $K(i-1)$, we see that (17) is true, which completes the proof.

From our last corollary we obtain a new proof of a well known property of algebraic shifting, proved by Kalai [4]:

Corollary $5.7 \quad \Delta^{2}=\Delta$.

Proof: For every simplicial complex $K$ with $n$ vertices, $\Delta(K)$ is shifted, (hence an $n$-near cone), and so are all the $1 \mathrm{k}(i,(\Delta K)(i-1))$ 's associated to it. By induction on the number of vertices, $\Delta(\operatorname{lk}(i,(\Delta K)(i-1)))=\operatorname{lk}(i,(\Delta K)(i-1))-i$ for all $1 \leq i \leq n$. Thus, applying Corollary 5.6 to the $n$-near cone $\Delta(K)$, we get $\Delta(\Delta(K))=\Delta(K)$.

\section{Shifting join of simplicial complexes}

Let $K, L$ be two disjoint simplicial complexes (they include the empty set), and denote their join by $K * L$, i.e.

$$
K * L=\{S \cup T: S \in K, T \in L\} .
$$

$K * L$ is also a simplicial complex, and using the Künneth theorem with field coefficients (see [7], Theorem 58.8 and ex. 3 on p. 373) we can describe its homology in terms of the homologies of $K$ and $L$ :

$$
H_{i}(K \times L) \cong \bigoplus_{k+l=i} H_{k}(K) \otimes H_{l}(L)
$$

and

$$
0 \rightarrow \tilde{H}_{p+1}(K * L) \rightarrow \tilde{H}_{p}(K \times L) \rightarrow \tilde{H}_{p}(K) \oplus \tilde{H}_{p}(L) \rightarrow 0 .
$$


Recalling that $\beta_{i}(K)=|\{S \in \Delta(K):|S|=i+1, S \cup 1 \notin \Delta(K)\}|$ (Björner and Kalai [1]), we get a description of the number of faces in $\Delta(K * L)_{i}$ which after union with $\{1\}$ are not in $\Delta(K * L)$, in terms of numbers of faces of that type in $\Delta(K)$ and $\Delta(L)$. In particular, if the dimensions of $K$ and $L$ are strictly greater than 0 , the Künneth theorem implies:

$$
\beta_{\operatorname{dim}(K * L)}(K * L)=\beta_{\operatorname{dim}(K)}(K) \beta_{\operatorname{dim}(L)}(L)
$$

and hence

$$
\begin{gathered}
|\{S \in \Delta(K * L): 1 \notin S,|S|=\operatorname{dim}(K * L)+1\}|=\mid\{S \in \Delta(K): 1 \notin S, \\
|S|=\operatorname{dim}(K)+1\}|\times|\{S \in \Delta(L): 1 \notin S,|S|=\operatorname{dim}(L)+1\} \mid .
\end{gathered}
$$

We now show that more can be said about the faces of maximal size in $K * L$ that represent homology of $K * L$, i.e. proving Theorem 1.3.

Proof of Theorem 1.3: For a generic $f=\sum_{v \in K_{0} \cup L_{0}} \alpha_{v} e_{v}$ decompose $f=f(K)+f(L)$ with supports in $K_{0}$ and $L_{0}$ respectively. Denote $\operatorname{dim}(K)=k, \operatorname{dim}(L)=l, \operatorname{sod} \operatorname{dim}(K * L)=$ $k+l+1$. Observe that $\left(f(K)\left\lfloor(K * L)_{k+l+1}\right) \cap\left(f(L)\left\lfloor(K * L)_{k+l+1}\right)=\{0\}\right.\right.$. Denote by $f\left\lfloor(K)\right.$ the corresponding generic boundary operation on $\operatorname{span}\left\{e_{S}: S \in K\right\}$, and similarly for $L$. Looking at $\bigwedge(K * L)$ as a tensor product $(\bigwedge K) \otimes(\bigwedge L)$ we see that $\operatorname{Ker}_{k+l+1} f(K) \downarrow$ equals $\operatorname{Ker}_{k} f(K)\left\lfloor\left.\right|_{\wedge K} \otimes \bigwedge^{1+l} L\right.$, and also $\operatorname{Ker}_{k} f(K)\left\lfloor\left.\right|_{\wedge K} \cong \operatorname{Ker}_{k} f\lfloor(K)\right.$, and similarly when changing the roles of $K$ and $L$. Hence, we get

$$
\operatorname{Ker}_{k+l+1} f\left\lfloor=\operatorname{Ker}_{k+l+1} f(K)\left\lfloor\cap \operatorname { K e r } _ { k + l + 1 } f ( L ) \left\lfloor\cong \operatorname { K e r } _ { k } f \left\lfloor(K) \otimes \operatorname{Ker}_{l} f\lfloor(L) .\right.\right.\right.\right.
$$

For the first $i$ generic $f_{j}$ 's, by the same argument, we have:

$$
\begin{aligned}
\bigcap_{j \in[i]} \operatorname{Ker}_{k+l+1} f_{j} L & =\bigcap_{j \in[i]} \operatorname{Ker}_{k+l+1} f_{j}(K)\left\lfloor\cap \bigcap_{j \in[i]} \operatorname{Ker}_{k+l+1} f_{j}(L)\llcorner\right. \\
& \cong \bigcap_{j \in[i]} \operatorname{Ker}_{k} f_{j}\left\lfloor(K) \otimes \bigcap_{j \in[i]} \operatorname{Ker}_{l} f_{j}\lfloor(L) .\right.
\end{aligned}
$$

By Propositions 3.1 and 3.2 we get the claimed Eq. (2).

Remark For symmetric shifting, the assertion analogous to Theorem 1.3 is false. As an example, let each of $K$ and $L$ consist of three points. Thus, $K * L=K_{3,3}$ is the complete bipartite graph with 3 vertices on each side. By Theorem 1.3, $\{3,4\} \in \Delta\left(K_{3,3}\right)$, but $\{3,4\} \notin \Delta^{\text {symm }}\left(K_{3,3}\right)$ where $\Delta^{\text {symm }}$ stands for the symmetric shifting operator ([6], p. 128).

We now deal with the conjecture ([6], Problem 12)

$$
\Delta(K * L)=\Delta(\Delta(K) * \Delta(L))
$$


We give a counterexample showing that it is false even if we assume that one of the complexes $K$ or $L$ is shifted. Denote by $\Sigma K$ the suspension of $K$, i.e. the join of $K$ with the (shifted) simplicial complex consisting of two points.

Example Let $B$ be the graph consisting of two disjoint edges. In this case $\Delta(\Sigma(B)) \backslash$ $\Delta(\Sigma(\Delta((B)))=\{\{1,2,6\}\}$ and $\Delta(\Sigma(\Delta(B))) \backslash \Delta(\Sigma(B))=\{\{1,3,4\}\}$, so (surprisingly) we even get that

$$
\Delta(\Sigma(B))<_{L} \Delta(\Sigma(\Delta(B))),
$$

where the lexicographic partial order on simplicial complexes is defined (as in [6]) by: $K \leq_{L} L$ iff for all $r>0$ the lexicographically first $r$-face in $K \triangle L$ (if exists) belongs to $K$.

Conjecture 6.1 For any simplicial complex $K: \Delta(\Sigma(K)) \leq_{L} \Delta(\Sigma(\Delta(K)))$.

This manuscript was first put on the math arXiv about two years ago. Very recently Satoshi Murai announced a proof of Conjecture 6.1, and more generally, that $\Delta(K * L) \leq_{L} \Delta(\Delta(K) *$ $\Delta(L))$ for any two simplicial complexes $K$ and $L$.

Conjecture 6.2 (Topological invariance.) Let $K_{1}$ and $K_{2}$ be triangulations of the same topological space. Then $\Delta\left(\Sigma\left(K_{1}\right)\right)<_{L} \Delta\left(\Sigma\left(\Delta_{(}\left(K_{1}\right)\right)\right)$ iff $\Delta\left(\Sigma\left(K_{2}\right)\right)<_{L} \Delta\left(\Sigma\left(\Delta\left(K_{2}\right)\right)\right)$.

It would be interesting to find out when Eq. (20) holds. If both $K$ and $L$ are shifted, it trivially holds as $\Delta^{2}=\Delta$. By the remark to Corollary 5.4 it also holds if $K$ is a complete simplicial complex.

\section{Acknowledgments}

I would like to express my profound thanks to my supervisor Prof. Gil Kalai, for numerous helpful discussions. I deeply thank Isabella Novik for valuable remarks on early versions of this manuscript. Thanks go also to the referees; one of them deserves special thanks for a very careful reading and very helpful suggestions.

\section{References}

1. A. Björner and G. Kalai, "An extended Euler-Poincaré theorem," Acta Math. 161 (1988), 279-303.

2. G. Kalai, "A characterization of $f$-vectors of families of convex sets in $\mathbb{R}^{d}$, Part 1: Necessity of Eckhoff's conditions," Israel J. Math. 48 (1984), 175-195.

3. G. Kalai, "Hyperconnectivity of graphs," Graphs and Combi. 1 (1985), 65-79.

4. G. Kalai, "Symmetric matroids," J. Comb.Th.B. 50 (1990), 54-64.

5. G. Kalai, "The diameter of graphs of convex polytopes and $f$-vector theory," in: "Applied Geometry and Discrete Mathematics" DIMACS Series in Discrete Math. Comp. Sci. 4 (1991), 387-411.

6. G. Kalai, "Algebraic shifting," Adv. Stud. in Pure Math. 33 (2002), 121-163.

7. J. Munkres, Elements of algebraic topology, Addison-Wesley, Menlo Park, Calif. 1984.

8. K.S. Sarkaria, "Exterior shifting," Res. Bulletin of the Punjab University 43 (1993), 259-268. 